\title{
Signal Detection for OFDM-IDMA Uplink over Doubly Selective Channels
}

\author{
Tao Peng ${ }^{1}$, Yue Xiao ${ }^{1}$, Shaoqian $\mathrm{Li}^{1}$, Huaqiang Shu ${ }^{2}$, Eric Pierre Simon ${ }^{2}$ \\ ${ }^{1}$ Nation Key Lab of Sci. and Techno. On Commun. University of Electronic Sci. and Techno. of China, Chengdu, China \\ ${ }^{2}$ IEMN Lab, TELICE Group, University of Lille, Lille, France
}

Received June, 2013

\begin{abstract}
Orthogonal frequency division multiplexing-interleave division multiple access (OFDM-IDMA) systems may suffer from serious inter-carrier interference (ICI) in time-and frequency-selective (doubly selective) channels. In such case, the conventional OFDM-IDMA detection algorithm for quasi-static channels will result in significantly performance degradation. In this paper, signal detection is investigated for OFDM-IDMA uplink over doubly selective channels. Firstly, the impact of time-varying channels for OFDM-IDMA uplink is analyzed, which leads to the failure of the conventional algorithm. Secondly, a novel iterative detection algorithm is developed based on an integrated interference canceller, which can iteratively estimate and mitigate the ICI as well as multiple access interference (MAI) simultaneously. In addition, an improved detection algorithm is derived for reducing the complexity using an approximation to the mean and variance of the interference. Simulation results indicate that the proposed algorithm can significantly enhance the system performance to the conventional case, and the improved algorithm can strike a balance between performance and complexity.
\end{abstract}

Keywords: Interleave Division Multiple Access (IDMA); Orthogonal Frequency Division Multiplexing (OFDM); Doubly Selective Channel

\section{Introduction}

As a kind of non-orthogonal multiple access scheme, interleave division multiple access (IDMA) was developed by Ping et al.[1,2], in which random interleavers are employed as the only means for user separation. In general, IDMA outperforms conventional code division multiple access (CDMA) in terms of power and bandwidth efficiency. The key innovation of IDMA is the introduction of low-rate channel coding, chip-level interleaving and low-complexity multiuser detection. On the other hand, orthogonal frequency division multiplexing (OFDM) is an attractive transmission technique for future wireless communication due to its high spectral efficiency and excellent robustness to frequency-selective fading. Based on the combination of IDMA and OFDM, OFDM-IDMA was presented for achieving the advantages of both systems [3]. In OFDM-IDMA systems, inter-symbol interference (ISI) can be mitigated by OFDM and multiple

\footnotetext{
"This work was supported by the Foundation Project of National Key Laboratory of Science and Technology on Communications under Grant 9140C020404120C0201, National High-Tech R\&D Program of China ("863" Project under Grant number 2011AA01A105), National Grand Special Science and Technology Project of China under Grant No. 2010ZX03006-002-02, and the Fundamental Research Funds for the Central Universities.
}

access interference (MAI) can be suppressed by IDMA. Moreover, OFDM-IDMA can achieve more throughput and reliability than conventional OFDM-CDMA and orthogonal frequency-division multiple access (OFDMA) [3-5].

In [3], an iterative multi-user detection scheme is derived for OFDM-IDMA, where the complexity of each user is independent of the channel length and number of users. However, it assumes that the frequency-selective channels are time invariant (quasi-static) within an OFDM block. In practice, high speed movement of mobile terminals will cause Doppler spread and result in timevarying multipath fading channels [6,7]. In this case, the channels will be time and frequency-selective (doubly selective), in which the length of an OFDM block is longer than the coherent time. As a result, the time variation of doubly selective channels will destroy the orthogonal property among subcarriers and induce inter-carrier interference (ICI), which complicates the data detection in the receiver.

Due to this additional interference from other subcarriers, the conventional OFDM-IDMA detection algorithm [3] will degrade the system performance severely in the doubly selective channels. In [8-10], several intercarrier interference cancellation schemes were proposed 
for OFDMA and OFDM-CDMA in doubly selective channels. However, due to different system structure, these schemes cannot be extended to OFDM-IDMA. Therefore, signal detection of OFDM-IDMA uplink over doubly selective channels becomes a challenging problem due to the complex interference from MAI and ICI.

In order to overcome the above problems over the doubly selective channels, signal detection for OFDMIDMA uplink is investigated in this paper. Firstly, the impact of doubly selective channels for OFDM-IDMA uplink is analyzed, which results in the failure of the conventional algorithm. Then a new iterative detection algorithm is developed based on an integrated interference canceller, which considers the influence of not only multiple users but also intercarrier interference. It can eliminate ICI from other subcarriers as well as MAI in an iterative way. Moreover, an improved detection algorithm is also derived based on an approximation to the mean and variance of the interference for reducing the complexity. Finally, simulation results verify that the proposed detection algorithm can greatly improve the performance of the conventional one, and the improved algorithm can achieve performance close to the proposed one while keeping the computational complexity low.

The rest of this paper is organized as follows. Section II introduces the system model of OFDM-IDMA uplink adopted in this paper. In Section III, three detection algorithms are presented for OFDM-IDMA uplink over doubly selective channels. Simulation results are provided in Section IV to demonstrate the effectiveness of the proposed algorithms. The conclusions are drawn in Section V.

\section{System Model}

\subsection{Transmitter of OFDM-IDMA Uplink}

The system model of OFDM-IDMA uplink with $K$ simultaneous users is shown in Figure 1, where each user communicates with the base station through independent doubly selective channels. For the transmitter of user- $k$, information data $d_{k}$ is first encoded and interleaved to generate a low-rate permutated sequence $X_{k}$, which is then fed into an inverse fast Fourier transform (IFFT) modulator. After IFFT and cyclic prefix (CP) insertion, the time domain transmitted signal from user- $k$ can be represented as

$$
X_{k}(n)=\frac{1}{N} \sum_{u=0}^{N-1} X_{k}(u) e^{j 2 \pi u n / N},-N_{g} \leq n \leq N-1
$$

where $N$ is the total number of subcarriers and $N_{g}$ is the length of $\mathrm{CP}$.

\subsection{Receiver of OFDM-IDMA Uplink}

As illustrated in Figure 1, the OFDM-IDMA receiver consists of one elementary signal estimator (ESE) detector and $K$ soft-input soft-output (SISO) decoders (DECs), which are used to solve the multiple access channel constraint and the coding constraint respectively. The outputs of ESE and DECs are the extrinsic log-likelihood ratios (LLRs), which are updated via the interleavers and deinterleavers iteratively. More detailed description of OFDM-IDMA receiver can be found in [3].

\section{Detection Algorithms of OFDM-IDMA Uplink over Doubly Selective Channels}

In the OFDM-IDMA receiver, since the SISO DECs are standard a posteriori probability (APP) decoding, the distinction between different detection algorithms lies in the ESE detector. In what follows, three different ESE detection algorithms will be given for OFDM-IDMA uplink over doubly selective channels.

\subsection{Impact of Doubly Selective Channels for OFDM-IDMA}

The doubly selective channel model considered in this paper is the time-varying multipath fading channel with a delay spread $L$. The channel may vary within each OFDM block, depending on the velocity of the user's motion.

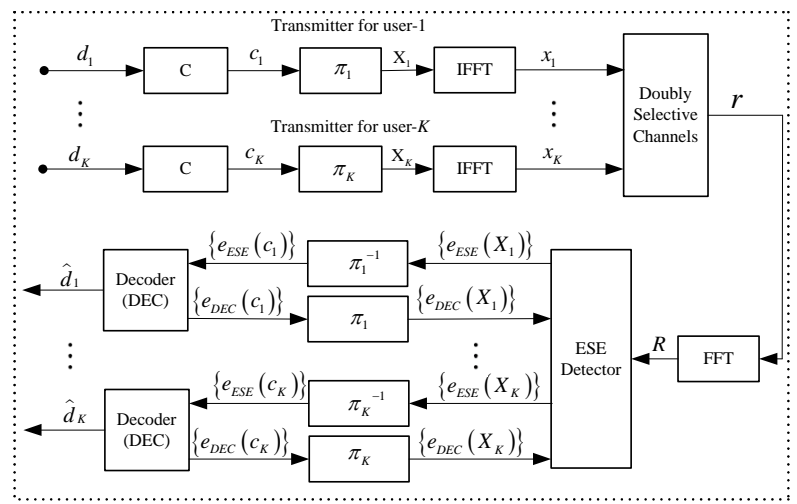

Figure 1. Transmitter and receiver structures of OFDM-IDMA uplink. 
The received signal from user- $k$ without noise can be described as

$$
\begin{aligned}
r_{k}(n) & =\sum_{l=0}^{L-1} h_{k}(n, l) x_{k}(n-l) \\
& =\frac{1}{N} \sum_{u=0}^{N-1} X_{k}(u) H_{k}(n, u) e^{j 2 \pi u n / N}
\end{aligned}
$$

where $h_{k}(n, l)$ stands for the channel impulse response (CIR) of path $l$ at time $n$ for user- $k$, and $H_{k}(n, u)$ is the channel frequency response at the $u$ th subcarrier.

Equation (2) can be rewritten in a matrix form as

$$
\mathbf{r}_{k}=\mathbf{H}_{k}^{t} \mathbf{X}_{k}
$$

where $\mathbf{r}_{k}=\left[r_{k}(0), r_{k}(1), \ldots, r_{k}(N-1)\right]^{T}, \mathbf{X}_{k}=\left[X_{k}(0), X_{k}(1), \ldots\right.$, $\left.X_{k}(N-1)\right]^{T}$, and $\mathbf{H}_{k}^{t}$ is given by (4), shown at the bottom of the page.

Thus the received signal from all users can be given by

$$
\mathbf{r}=\sum_{k=1}^{K} \mathbf{r}_{k}+\mathbf{w}=\sum_{k=1}^{K} \mathbf{H}_{k}^{t} \mathbf{X}_{k}+\mathbf{w}
$$

where $\mathbf{w}$ is the additive white Gaussian noise (AWGN) with zero mean and variance $\sigma^{2}$.

After the CP removal and fast Fourier transform (FFT) of $\mathbf{r}$ in (5), the received signal in the frequency domain is as follows

$$
\mathbf{R}=\mathbf{F}_{N} \mathbf{r}=\sum_{k=1}^{K} \mathbf{F}_{N} \mathbf{H}_{k}^{t} \mathbf{X}_{k}+\mathbf{W}
$$

where $\mathbf{W}$ is the noise in the frequency domain, and $\mathbf{F}_{N}$ is the $N \times N$ FFT matrix defined as

$$
\mathbf{F}_{N}(m+1, n+1)=e^{-j 2 \pi m n / N}, 0 \leq m, n \leq N-1 .
$$

Furthermore, denoting $\mathbf{F}_{N} \mathbf{H}_{k}^{t}$ by $\mathbf{H}_{k}^{f}$ in (6), the received signal can be rewritten as

$$
\mathbf{R}=\sum_{k=1}^{K} \mathbf{H}_{k}^{f} \mathbf{X}_{k}+\mathbf{W}
$$

In (8), $\mathbf{H}_{k}^{f}$ is the equivalent channel response matrix of the doubly selective channel for user- $k$.

\subsection{Conventional Detection Algorithm}

In [3], the conventional detection algorithm of OFDMIDMA was proposed, which can effectively cancel the MAI of quasi-static channels. When the channel is assumed to be time invariant within an OFDM block, the channel matrix $\mathbf{H}_{k}^{f}$ in (8) can be simplified to a diagonal matrix denoted as $\widetilde{H}_{k}^{f}$. In this case, the frequency domain received signal (8) is simplified as

$$
R_{\text {static }}(m)=\sum_{k=1}^{K} \widetilde{H}_{k}^{f}(m) X_{k}(m)+W(m), 0 \leq m \leq N-1 \text {. }
$$

For the subcarrier $m$ of user- $k$, the received signal can be rewritten as

$$
\begin{aligned}
R_{\text {static }}(m) & =\widetilde{H}_{k}^{f}(m) X_{k}(m)+\sum_{k^{\prime}=1, k^{\prime} \neq k}^{K} \widetilde{H}_{k^{\prime}}^{f}(m) X_{k^{\prime}}(m)+W(m) \\
& =\widetilde{H}_{k}^{f}(m) X_{k}(m)+\zeta_{k}^{M A I}(m)
\end{aligned}
$$

where $\zeta_{k}^{\text {MAI }}(m)$ is the total interference term with respect to user- $k$ on subcarrier- $m$, consisted of both MAI and noise.

Without loss of generality, BPSK modulation and realvalued channel coefficients are assumed for simplifying the expression. Furthermore, the principle here can be easily extended to other cases such as complex channels, higher order modulations and multiple receive antennas [3]. According to the central limit theorem, $\zeta_{k}^{\text {MAI }}(m)$ is approximated as a Gaussian random variable with mean $E\left(\zeta_{k}^{M A I}(m)\right)$ and variance $\operatorname{Var}\left(\zeta_{k}^{M A I}(m)\right)$. Based on the definition of extrinsic LLRs, the output of ESE detector is calculated by

$$
e_{E S E}\left(X_{k}(m)\right)=2 \widetilde{H}_{k}^{f}(m) \frac{R_{\text {static }}(m)-E\left(\zeta_{k}^{M A I}(m)\right)}{\operatorname{Var}\left(\zeta_{k}^{M A I}(m)\right)}
$$

where $E\left(\zeta_{k}^{\text {MAI }}(m)\right)$ and $\operatorname{Var}\left(\zeta_{k}^{\text {MAI }}(m)\right)$ can be obtained by the mean and variance of $X_{k},(m)$.

By the priori LLRs $e_{D E C}\left(X_{k}\right)$ feedback from the DECs, the mean and variance of $X_{k}(m)$ can be calculated as follows

$$
\begin{gathered}
E\left(X_{k}(m)\right)=\tanh \left(e_{D E C}\left(X_{k}(m)\right) / 2\right), \\
\operatorname{Var}\left(X_{k}(m)\right)=1-\left(E\left(X_{k}(m)\right)\right)^{2} .
\end{gathered}
$$

In the above conventional detection algorithm, the MAI $\zeta_{k}^{\text {MAI }}(m)$ of quasi-static channels can be eliminated effectively. However, in the doubly selective channel, the channel matrix $\mathbf{H}_{k}^{f}$ is not a diagonal matrix and the frequency domain received signal cannot be expressed

$$
\mathbf{H}_{k}^{t}=\frac{1}{N}\left(\begin{array}{cccc}
H_{k}(0,0) & H_{k}(0,1) & \cdots & H_{k}(0, N-1) \\
H_{k}(1,0) & H_{k}(1,1) e^{\frac{j 2 \pi}{N}} & \cdots & H_{k}(1, N-1) e^{\frac{j 2 \pi(N-1)}{N}} \\
\vdots & \vdots & \ddots & \vdots \\
H_{k}(N-1,0) & H_{k}(N-1,1) e^{\frac{j 2 \pi(N-1)}{N}} & \cdots & H_{k}(N-1, N-1) e^{\frac{j 2 \pi(N-1)^{2}}{N}}
\end{array}\right)
$$


simply as (9). More specifically, an additional interference from other subcarriers will be introduced, resulting from the time-varying characteristics of the channel. Therefore, the performance will be degraded significantly if the conventional algorithm is adopted in the doubly selective channels.

\subsection{Proposed Detection Algorithm}

To alleviate this problem, a novel ESE detection algorithm is proposed to enhance the system performance of OFDM-IDMA uplink over doubly selective channels. It takes into account the additional intercarrier interference and utilizes an iterative soft interference canceller to suppress the MAI and ICI jointly. With increasing iteration number, the additional interference can be mitigated and the transmitted signal of the desired user can be recovered gradually.

For analyzing the interference of user- $k$, the received signal on subcarrier- $m$ in (8) is expressed as

$$
\begin{aligned}
R(m) & =\sum_{k=1}^{K} \sum_{n=0}^{N-1} H_{k}^{f}(m, n) X_{k}(n)+W(m) \\
& =H_{k}^{f}(m, m) X_{k}(m)+\sum_{k=1}^{K} \sum_{n=0, n \neq m}^{N-1} H_{k}^{f}(m, n) X_{k}(n)+\zeta_{k}^{\text {MAI }}(m) \\
& =H_{k}^{f}(m, m) X_{k}(m)+\zeta_{k}^{\text {III }}(m)+\zeta_{k}^{\text {MAI }}(m) .
\end{aligned}
$$

As shown in (14), the interference term includes not only the MAI from other users on subcarrier- $m$, but also the ICI from other subcarrier- $n(n \neq m, 0 \leq n \leq N-1)$ of all users. Compared to (10), an additional interference term $\zeta_{k}^{\text {ICI }}(m)$ will be appeared in the doubly selective channels, which is neglected in the conventional algorithm. This explains the performance degradation for OFDM-IDMA uplink when using the conventional algorithm. Consequently, this additional interference should also be cancelled in the ESE detector. Based on an integrated interference canceller, the output of the proposed ESE detector can be obtained by

$$
\begin{aligned}
& e_{E S E}^{\text {proposed }}\left(X_{k}(m)\right) \\
= & 2 H_{k}^{f}(m, m) \frac{R(m)-\left(E\left(\zeta_{k}^{\text {MAI }}(m)\right)+E\left(\zeta_{k}^{I C I}(m)\right)\right)}{\operatorname{Var}\left(\zeta_{k}^{\text {MAI }}(m)\right)+\operatorname{Var}\left(\zeta_{k}^{I C I}(m)\right)} .
\end{aligned}
$$

In the proposed detection algorithm, all the interference included MAI and ICI can be suppressed and the system performance can be improved. For suppressing the additional interference $\zeta_{k}^{\text {ICI }}(m)$, the computation in the proposed detection algorithm is more complicated than the conventional one.

\subsection{Improved Low-Complexity Detection Algorithm}

As described above, the proposed detection algorithm can obtain more satisfied performance than the conventional one. But the computation complexity of the mean and variance of the interference is much higher than the conventional one. In the follow, an improved detection algorithm is proposed for reducing the complexity by an approximation to the mean and variance of the interference.

In the proposed algorithm, the interference $\zeta_{k}^{I C I}(m)$ consists of the equivalent channel matrix $\mathbf{H}_{k}^{f}$ and the transmitted signal on other subcarriers. This indicates that the value of the channel matrix $\mathbf{H}_{k}^{f}$ will affect the computation of the mean and variance of $\zeta_{k}^{I C I}(m)$. By analyzing the property of the channel matrix $\mathbf{H}_{k}^{f}$, it can be found that the values of off-diagonal elements become smaller and smaller with increasing distance to the main diagonal elements. And when these off-diagonal elements are far from the main diagonal ones, their values will be quite small that can be approximated to zero. As a result, the row- $m(1 \leq m \leq N)$ of the channel matrix $\mathbf{H}_{k}^{f}$ ( $1 \leq k \leq K)$ can be approximated by using the following vector:

$$
\begin{aligned}
\widehat{H}_{k}^{f}(m,:)= & {\left[0, \ldots, 0, H_{k}^{f}(m, m-(P-1) / 2), \ldots, H_{k}^{f}(m, m),\right.} \\
& \left.\ldots, H_{k}^{f}(m, m+(P-1) / 2), 0, \ldots, 0\right]
\end{aligned}
$$

where $P$ is the number of elements that are not set to zero. In this case, the interference $\zeta_{k}^{\text {ICI }}$ only from $P$ subcarriers needs to be calculated instead of all subcarriers in the ESE detector.

Furthermore, by the expression in (15), the variance of interference only affects the magnitude of the output of ESE detector. Thus the variance of $\zeta_{k}^{\text {ICI }}(m)$ can be neglected for reducing the computation complexity.

Consequently, the improved algorithm can reduce the complexity in two aspects. Firstly, the computation complexity of the mean of the interference is reduced to $P / N$ as the proposed algorithm by the approximation to the channel matrix. Secondly, the computation complexity of the variance of the interference is reducing to the same as the conventional one.

\section{Simulation Results}

In this section, computer simulations are carried out to verify the effectiveness of the proposed detection algorithms over doubly selective channels. Simulations are performed for OFDM-IDMA uplink with four users, employing BPSK modulation. A repetition code of rate-1/8 is adopted for all users, and the total number of subcarriers is 128. The channel model used here is the extended vehicular A (EVA) channel [11]. The normalized Doppler frequency is denoted as $f_{D} T$, where $f_{D}$ represents the maximum Doppler frequency shift and $T$ is an OFDM symbol period. In the simulations, channel estimation is not considered and perfect channel information is assumed to be known at the receiver.

Figure 2 shows the bit-error-rate (BER) performance of the conventional detection algorithm in the doubly 
selective channels with different velocities (i.e., correspondingly $f_{D} T=0.001,0.01,0.05,0.1$, and 0.2 , respectively). It can be found that the system performance degrades with the increase of users' velocity. The performance is relatively poor at $f_{D} T=0.2$ and $f_{D} T=0.1$ compared with $f_{D} T=0.01$. This is because the conventional algorithm doesn't take into account the additional interference from other subcarriers, which becomes larger with the increase of normalized Doppler frequency.

Figure 3 depicts the BER performance results of the proposed and conventional detection algorithms in the doubly selective channels with different normalized Doppler shift. From this figure, it can be found that the proposed algorithm can enhance the performance obviously in the doubly selective channels. More specifically, it can be found that the performance gap between the proposed algorithm and the conventional one is very significant when $f_{D} T=0.2$. And the performance of the proposed algorithm with $f_{D} T=0.1$ is very close to the performance of the conventional one with $f_{D} T=0.001$.

Figure 4 and Figure 5 show the BER performance of the improved low-complexity algorithm with different value of parameter $P$, where the performance of the conventional and proposed algorithms is also included for comparison. It can be observed that the performance gap between the improved algorithm and conventional one becomes greater with the increasing value of $P$. When $P$ $=3$, at the cost of little complexity, the performance of the improved algorithm is significantly better than that of the conventional one. And while $P=17$ for the improved algorithm, the performance is almost the same as that of the proposed one.

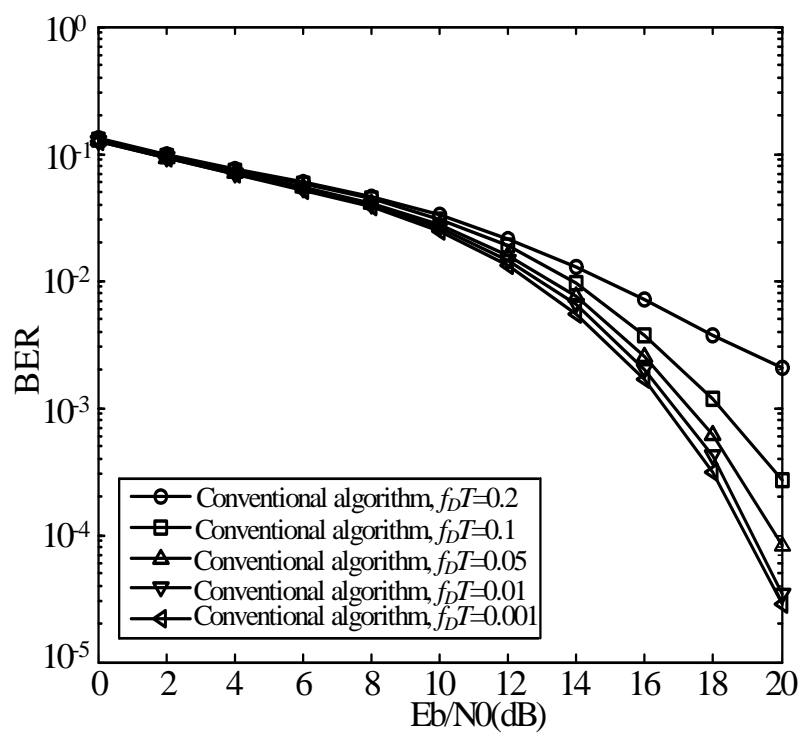

Figure 2. BER performance of the conventional algorithm in the doubly selective channels with different normalized Doppler frequency $f_{D} T$.

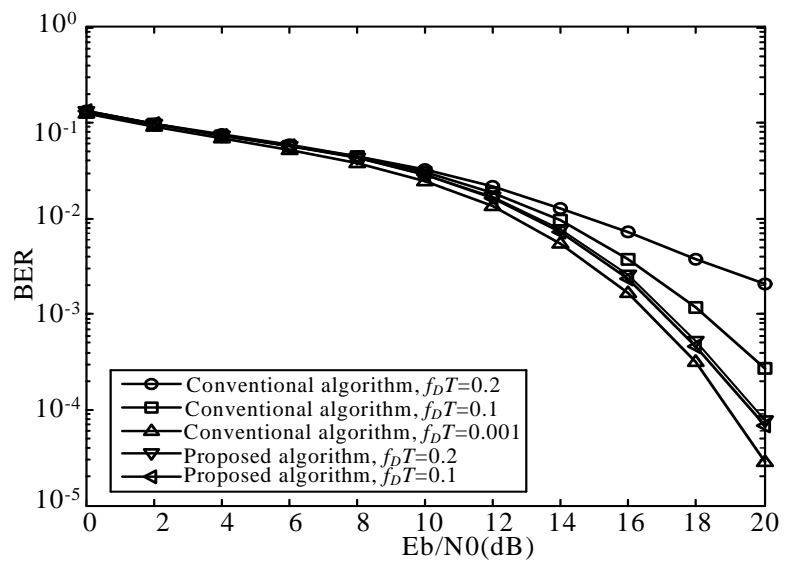

Figure 3. Performance comparison of the proposed algorithm and the conventional one in the doubly selective channels with different normalized Doppler frequency $f_{D} T$.

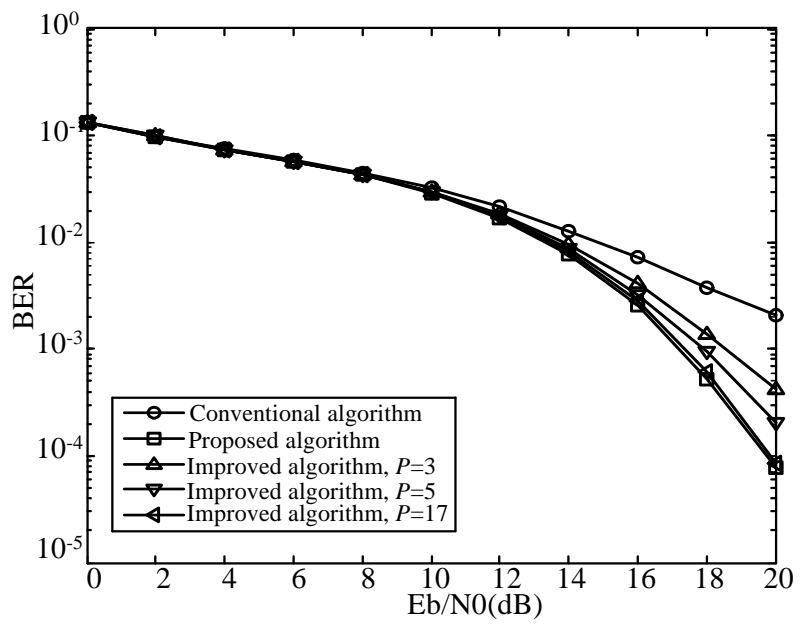

Figure 4. BER performance of the improved algorithm in the doubly selective channels $\left(f_{D} T=0.2\right)$ with different parameter $P$.

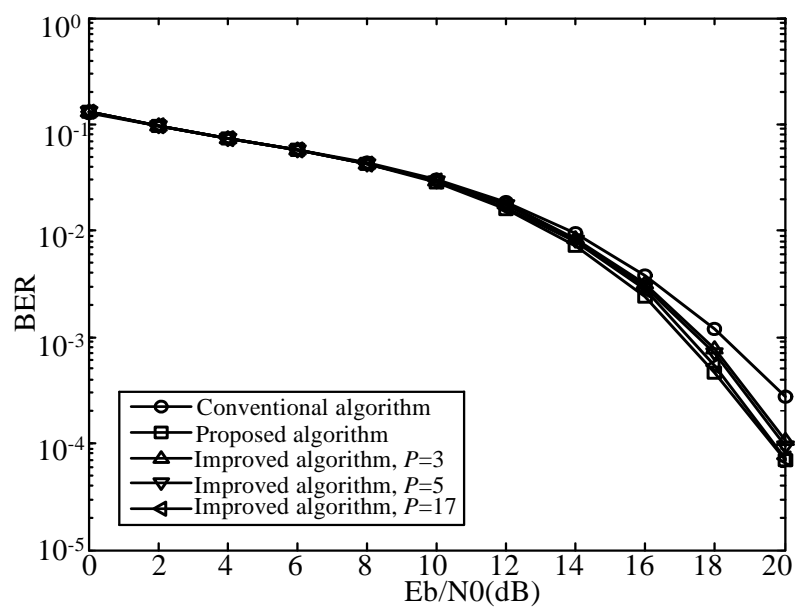

Figure 5. BER performance of the improved algorithm in the doubly selective channels $\left(f_{D} T=0.1\right)$ with different parameter $\boldsymbol{P}$. 


\section{Conclusions}

In this paper, three different detection algorithms are derived for OFDM-IDMA uplink over doubly selective channels. Among all the three detection algorithms, the conventional algorithm suffers from performance loss due to neglect of the additional interference from other subcarriers, and the proposed algorithm delivers the best performance but at the cost of higher complexity. And the improved algorithm can achieve performance close to the proposed one but with a low computational complexity. Consequently, it is optimal to adopt the improved lower-complexity algorithm to obtain a tradeoff between performance and complexity.

\section{REFERENCES}

[1] P. Li, L. H. Liu, K. Y. Wu and W. K. Leung, “A Unified Approach to Multiuser Detection and Space-time Coding with Low Complexity and Nearly Optimal Performance,” in Proc. 40th Allerton Conference, 2002, pp. 170-179.

[2] P. Li, L. H. Liu, K. Y. Wu and W. K. Leung, "Interleave Division Multiple Access," IEEE Transactions Wireless Communications, Vol. 5, No. 4, 2006, pp. 938-947.

[3] P. Li, Q. H. Guo and J. Tong, "The OFDM-IDMA Approach to Wireless Communication Systems," IEEE Wireless Communications Mag., Vol. 14, No. 3, 2007, pp. 18-24.

[4] R. Zhang and L. Hanzo, "Three Design Aspects of Multicarrier Interleave Division Multiple Access," IEEE Transactions Vehicular Technoogy, Vol. 57, No. 6, 2008, pp. 3607-3617. doi:10.1109/TVT.2008.918724

[5] P. Hammarberg, F. Rusek and O. Edfors, "Channel Estimation Algorithms for OFDM-IDMA: Complexity and Performance," IEEE Transactions Wireless Communications, Vol. 11, No. 5, 2012, pp. 1722-1732.

[6] Y. Li and L. J. Cimini, "Bounds on the Interchannel Interference of OFDM in Time-varying Impairments," IEEE Transactions Communications, Vol. 49, No. 3, 2001, pp. 401-404. doi:10.1109/26.911445

[7] P. Schniter, "Low-Complexity Equalization of OFDM in Doubly Selective Channels," IEEE Transactions Communications, Vol. 52, No. 4, 2004, pp. 1002-1011.

[8] Y. X. Peng, K. Zheng, W. Wang, Y. Kim and Y. S. Lee, "Iterative Partial-Interference-Cancellation-based Detector for OFDM Systems over Doubly-Selective Rayleigh Fading Channels," in Proc. IEEE International Symp. On Personal, Indoor and Mobile Radio Comm., 2007, pp. $1-5$.

[9] T. Zemen, C. F. Mecklenbrauker, J. Wehinger and R. R. Muller, "Iterative Joint Time-variant Channel Estimation and Multi-user Detection for MC-CDMA," IEEE Transactions Wireless Communications, Vol. 5, No. 4, 2006, pp. 938-947.

[10] S. W. Hou and C. C. Ko, "Intercarrier Interference Suppression for OFDMA Uplink in Time-and Frequency-Selective Fading Channels,” IEEE Transactions Vehicular Technoogy, Vol. 58, No. 6, 2009, pp. 2741-2754. doi:10.1109/TVT.2008.2010550

[11] 3GPP Technical Specification 36.104, 2010, “Evolved Universal Terrestrial Radio Access (E-UTRA): Base Station(BS) Radio Transmission and Reception," Version 9.3.0, Mar. 2010 